\title{
A method for inversion of layered shear wavespeed azimuthal anisotropy from Rayleigh wave dispersion using the Neighborhood Algorithm
}

\author{
Huajian Yao
}

Received: 22 October 2014/Accepted: 22 December 2014/Published online: 4 February 2015

(C) The Author(s) 2015. This article is published with open access at Springerlink.com

\begin{abstract}
Seismic anisotropy provides important constraints on deformation patterns of Earth's material. Rayleigh wave dispersion data with azimuthal anisotropy can be used to invert for depth-dependent shear wavespeed azimuthal anisotropy, therefore reflecting depth-varying deformation patterns in the crust and upper mantle. In this study, we propose a two-step method that uses the Neighborhood Algorithm (NA) for the point-wise inversion of depth-dependent shear wavespeeds and azimuthal anisotropy from Rayleigh wave azimuthally anisotropic dispersion data. The first step employs the NA to estimate depthdependent $V_{\mathrm{SV}}$ (or the elastic parameter $L$ ) as well as their uncertainties from the isotropic part Rayleigh wave dispersion data. In the second step, we first adopt a difference scheme to compute approximate Rayleigh-wave phase velocity sensitivity kernels to azimuthally anisotropic parameters with respect to the velocity model obtained in the first step. Then we perform the NA to estimate the azimuthally anisotropic parameters $G_{\mathrm{c}} / L$ and $G_{\mathrm{s}} / L$ at depths separately from the corresponding cosine and sine terms of the azimuthally anisotropic dispersion data. Finally, we compute the depth-dependent magnitude and fast polarization azimuth of shear wavespeed azimuthal anisotropy. The use of the global search NA and Bayesian analysis allows for more reliable estimates of depth-dependent shear wavespeeds and azimuthal anisotropy as well as their uncertainties.
\end{abstract}

H. Yao $(\bowtie)$

Laboratory of Seismology and Physics of Earth's Interior,

School of Earth and Space Sciences, University of Science and

Technology of China, Hefei, Anhui, China

e-mail: hjyao@ustc.edu.cn

H. Yao

National Geophysical Observatory at Mengcheng, Hefei, Anhui, China
We illustrate the inversion method using the azimuthally anisotropic dispersion data in SE Tibet, where we find apparent changes of fast axes of shear wavespeed azimuthal anisotropy between the crust and uppermost mantle.

Keywords Azimuthal anisotropy - Shear wavespeed · Rayleigh wave $\cdot$ Neighborhood Algorithm

\section{Introduction}

Surface wave tomography from earthquake data or ambient noise cross-correlations has been widely used to investigate crust and upper mantle shear wave velocity structures globally or regionally. A majority of surface wave studies have focused on the inversion of isotropic shear wavespeeds (e.g., Simons et al. 1999; Huang et al. 2003; Yao et al. 2008; An et al. 2009; Li et al. 2009; Zheng et al. 2010; Sun et al. 2010; Yang et al. 2012; Li et al. 2013), while some other studies focus on the inversion of radially or azimuthally anisotropic shear velocity structures. These studies are important for understanding shape or lattice preferred orientations of minerals and deformation styles in the crust and upper mantle (Savage 1999; Mainprice 2007; Montagner 2007). For instance, Shapiro and Ritzwoller (2002) and Zhou et al. (2006) used earthquake Rayleigh and Love waves to obtain global models of shear wavespeed radial anisotropy in the upper mantle, that is, the difference between the vertically polarized shear wavespeed $\left(V_{\mathrm{SV}}\right)$ and the horizontally polarized shear wavespeed $\left(V_{\mathrm{SH}}\right)$. Ambient noise tomography using both Rayleigh and Love waves can produce high-resolution shear wavespeed radial anisotropy in the crust (e.g., Huang et al. 2010; Moschetti et al. 2010; Guo et al. 2012; Luo et al. 2013). 
In regions with good azimuthal path coverage, perioddependent 2-D phase/group velocity maps with azimuthal anisotropy can be obtained either from direct inversion of phase/group velocity dispersion measurements (e.g., Montagner 1986; Su et al. 2008; Fry et al. 2010; Yao et al. 2010; Yi et al. 2010; Endrun et al. 2011; Lu et al. 2014) or from localized estimation of phase velocity and its azimuthal anisotropy by solving the Eikonal equation (Lin et al. 2009) or the Helmholtz equation (Lin and Ritzwoller 2011). Montagner and Nataf (1986) proposed a linearized inversion method for inverting azimuthal anisotropy of surface wave dispersion for shear wavespeeds with azimuthal and radial anisotropy at depths. This method has been used to obtain upper mantle azimuthal anisotropy regionally (e.g., Montagner and Jobert 1988; Silveira and Stutzmann 2002; Yao et al. 2010) and globally (e.g., Montagner and Tanimoto 1990). Another approach to obtain the depth-dependent $V_{\mathrm{SV}}$ and azimuthal anisotropy is following a two-step procedure based on waveform inversions: (1) nonlinear surface waveform inversion to obtain 1-D path-averaged $V_{\mathrm{SV}}$ models and (2) tomographic inversion to invert all 1-D path-averaged $V_{\mathrm{SV}}$ models for $3-\mathrm{D} V_{\mathrm{SV}}$ structures and azimuthal anisotropy (e.g., Simons et al. 2002; Debayle et al. 2005).

The inversion of (isotropic) shear wavespeed structure from surface wave dispersion is nonlinear and inversion results may depend on the initial reference model. A number of global optimization algorithms can be applied to invert dispersion data for shear wavespeed models, including the Monte-Carlo approach (Shapiro and Ritzwoller 2002), the Neighborhood Algorithm (Sambridge 1999a, b; Yao et al. 2008), the Genetic Algorithms (Shi and Jin 1995; Wu et al. 2001), etc. Instead of just giving one best-fit model in the linearized inversion approach, these global searching methods typically perform (quasi-) random walks in the model space, retain a subset of models that satisfy certain misfit criteria, and finally give an ensemble of acceptable models. From Bayesian statistical analysis of the generated model ensemble, we can access uncertainties of model parameters and correlations between different model parameters (e.g., Sambridge 1999b; Yao et al. 2008).

From Montagner and Nataf (1986), the inversion of azimuthally anisotropic parameters of shear wavespeeds depends on the isotropic part shear wavespeed structure. Therefore, a reliable isotropic shear wavespeed model is important for robust estimation of azimuthally anisotropic model parameters. In this study, we propose a two-step inversion method using the Neighborhood Algorithm and Bayesian analysis (Sambridge 1999a, b) to invert azimuthally anisotropic Rayleigh wave dispersion data for the 1-D model of depth-dependent shear wavespeed and azimuthal anisotropy. We will first describe the details of the proposed methodology and then apply this method in SE Tibet. Finally, we will discuss the proposed methodology and the inversion results.

\section{Methodology}

\subsection{Rayleigh wave azimuthal anisotropy}

From Smith and Dahlen (1973) Rayleigh-wave phase velocity $c(\omega, M, \psi)$ at location $M$ for an angular frequency $\omega$ and propagation azimuth $\psi$ (with respect to north) can be expressed as

$$
\begin{aligned}
c(\omega, M, \psi)= & c_{0}(\omega)+a_{0}(\omega, M)+a_{1}(\omega, M) \cos 2 \psi \\
& +a_{2}(\omega, M) \sin 2 \psi+a_{3}(\omega, M) \cos 4 \psi \\
& +a_{4}(\omega, M) \sin 4 \psi,
\end{aligned}
$$

where $c_{0}(\omega)$ is the reference phase velocity from a reference model, and $a_{0}$ is the isotropic phase velocity perturbation with respect to the reference phase velocity, $a_{1,2}$ and $a_{3,4}$ are the azimuthally anisotropic coefficients for the $2 \psi\left(180^{\circ}\right.$ periodicity) and $4 \psi\left(360^{\circ}\right.$ periodicity) terms, respectively. As noted by Montagner and Nataf (1986), the $4 \psi$ terms are negligibly small for Rayleigh waves. Therefore, by ignoring the $4 \psi$ terms in Eq. (1), the perturbation of phase velocity with respect to the reference $c_{0}(\omega)$ can be written as

$$
\begin{aligned}
\delta c_{\mathrm{R}}(\omega, M, \psi) \approx & a_{0}(\omega, M)+a_{\mathrm{c}}(\omega, M) \cos 2 \psi \\
& +a_{\mathrm{s}}(\omega, M) \sin 2 \psi,
\end{aligned}
$$

where $a_{\mathrm{c}}, a_{\mathrm{s}}$ are used here to replace $a_{1}, a_{2}$, respectively in Eq. (1) in order to denote the cosine and sine terms.

Following Montagner and Nataf (1986), we express the Rayleigh-wave phase velocity perturbation $\delta c_{\mathrm{R}}(M, \omega, \psi)$ at location $M$ as

$$
\begin{aligned}
\delta c_{\mathrm{R}}(M, \omega, \psi) \approx & \int_{0}^{H}\left[\frac{\partial c_{\mathrm{R}}}{\partial A}\left(\delta A+B_{\mathrm{c}} \cos 2 \psi+B_{\mathrm{s}} \sin 2 \psi\right)\right. \\
& +\frac{\partial c_{\mathrm{R}}}{\partial C} \delta C+\frac{\partial c_{\mathrm{R}}}{\partial F}\left(\delta F+H_{\mathrm{c}} \cos 2 \psi\right. \\
& \left.+H_{\mathrm{s}} \sin 2 \psi\right)+\frac{\partial c_{\mathrm{R}}}{\partial L}\left(\delta L+G_{\mathrm{c}} \cos 2 \psi\right. \\
& \left.\left.+G_{\mathrm{s}} \sin 2 \psi\right)\right] \mathrm{d} z
\end{aligned}
$$

The four parameters $(A, C, F, L)$ in (3) together with the other one $N$ describe the equivalent transversely isotropic medium with a vertical symmetry axis with $A=\rho V_{\mathrm{PH}}^{2}$, $C=\rho V_{\mathrm{PV}}^{2}, L=\rho V_{\mathrm{SV}}^{2}, N=\rho V_{\mathrm{SH}}^{2}$, in which $\rho$ is density, $V_{\mathrm{PH}}$ and $V_{\mathrm{PV}}$ are the horizontally and vertically 
"propagating" P-wave velocities, $V_{\mathrm{SH}}$ and $V_{\mathrm{SV}}$ are the horizontally and vertically "polarized" S-wave velocities, respectively. The other six parameters $B_{\mathrm{s}, \mathrm{c}}, G_{\mathrm{s}, \mathrm{c}}$, and $H_{\mathrm{s}, \mathrm{c}}$ give the $2 \psi$ azimuthal variations $\left(180^{\circ}\right.$ periodicity) of $A, L$, and $F$, respectively. The kernels $\partial c_{\mathrm{R}} / \partial p_{i}\left(p_{i}=A, L\right.$, or $F$ ) can be calculated from a 1-D reference model. A generalized least squares inversion approach can be implemented to solve the Eq. (3) to obtain these elastic parameters (Montagner and Nataf 1986).

Montagner and Nataf (1986) found that the $\partial c_{R} / \partial L$ term has the largest contribution in Eq. (3), $\partial c_{R} / \partial A$ is comparably large in the crust and negligibly small in the upper mantle, but $\partial c_{\mathrm{R}} / \partial F$ is somewhat smaller. Therefore, we can approximate (3) by ignoring the $\partial c_{\mathrm{R}} / \partial F$ term as

$$
\begin{aligned}
\delta c_{\mathrm{R}}(M, \omega, \psi) \approx & \int_{0}^{H}\left[\frac{\partial c_{\mathrm{R}}(\omega)}{\partial A}\left(\delta A+B_{\mathrm{c}} \cos 2 \psi+B_{\mathrm{s}} \sin 2 \psi\right)\right. \\
& +\frac{\partial c_{\mathrm{R}}(\omega)}{\partial L}\left(\delta L+G_{\mathrm{c}} \cos 2 \psi+G_{\mathrm{s}} \sin 2 \psi\right) \\
& \left.+\frac{\partial c_{\mathrm{R}}}{\partial C} \delta C\right] \mathrm{d} z
\end{aligned}
$$

Since for every azimuth $\psi$, (4) holds, thus we have

$a_{0}(\omega, M) \approx \int_{0}^{H}\left[\frac{\partial c_{\mathrm{R}}(\omega)}{\partial A} \delta A+\frac{\partial c_{\mathrm{R}}(\omega)}{\partial L} \delta L+\frac{\partial c_{\mathrm{R}}}{\partial C} \delta C\right] \mathrm{d} z$,

$a_{\mathrm{c}, \mathrm{s}}(\omega, M) \approx \int_{0}^{H}\left[\frac{\partial c_{\mathrm{R}}(\omega)}{\partial A} B_{\mathrm{c}, \mathrm{s}}+\frac{\partial c_{\mathrm{R}}(\omega)}{\partial L} G_{\mathrm{c}, \mathrm{s}}\right] \mathrm{d} z$

The use of the subscript c,s in (6) means there are two equations, one taking the subscript $\mathrm{c}$ and the other one taking $\mathrm{s}$ in all corresponding variables in (6). This notation will be similarly used hereinafter. Equation (5) can be used to solve for $\delta A, \delta L$, and $\delta C$ from the isotropic part of Rayleigh-wave phase velocity perturbations using the (iterative) linearized inversion method. However, usually only $\delta L$ can be well resolved due to large sensitivity of $\partial c_{\mathrm{R}} /$ $\partial L$ in (5) (Montagner and Nataf 1986).

\subsection{Inversion for shear wavespeeds and azimuthal anisotropy}

We can invert for the isotropic part $V_{\mathrm{SV}}$ of a layered model from dispersion data using global searching algorithms, for instance, Metropolis Monte-Carlo Algorithm (Shapiro and Ritzwoller 2002), Neighborhood Algorithm (Sambridge 1999a, b; Yao et al. 2008), etc. Typically we perform forward calculations of the isotropic part dispersion data $c^{\text {pred }}(\omega)$ from an ensemble of the generated models (a function of $V_{\mathrm{P}}, V_{\mathrm{S}}$ and $\rho)$, which are compared with the observed isotropic part dispersion measurements $c^{\text {obs }}(\omega)=c_{0}(\omega)+a_{0}(\omega, M)$ in order to obtain best fitting models.
In this study, we use the global searching Neighborhood Algorithm (NA) and Bayesian analysis (Sambridge 1999a, b) to estimate $L$ and $G_{\mathrm{c}, \mathrm{s}}$ as well as their uncertainties. The NA involves two stages: (1) the NAS stage (Sambridge 1999a), which consists of a model space search based on Voronoi cells to identify the "good" fitting model regions; and (2) the NAB stage (Sambridge 1999b), which employs the Bayesian statistical analysis of the generated model ensemble in the NAS stage to compute the posterior mean model parameters and their uncertainties from the 1-D marginal posterior probability density functions (1-D PPDFs or 1-D marginals) and trade-offs between different two model parameters from the 2-D PPDFs (or 2-D marginals).

From (6), we note that the reliable estimation of azimuthal anisotropy $\left(G_{\mathrm{s}, \mathrm{c}}\right.$ and $\left.B_{\mathrm{s}, \mathrm{c}}\right)$ relies on accuracy of the sensitivity kernels $\partial c_{\mathrm{R}} / \partial L$ and $\partial c_{\mathrm{R}} / \partial A$, therefore it is important to first obtain a good isotropic reference model to compute these kernels. Therefore, we propose a two-step inversion strategy:

(1) Step 1: Perform the NA to estimate the layered $V_{\mathrm{SV}}$ (or $L$ ) as well as their uncertainties from the isotropic part Rayleigh wave dispersion data $\left(c^{\text {obs }}(\omega)\right.$, at all available frequencies). We use the method due to Herrmann and Ammon (2004) to compute the dispersion for an isotropic model. $V_{\mathrm{PH}}($ or $A$ ) and $\rho$ are linked to $V_{\mathrm{SV}}$ (or $L$ ) using some empirical relationships in the crust (Brocher 2005) and upper mantle (Masters et al. 2000). We refer to Yao et al. (2008, 2010) for the details of this step.

(2) Step 2: Perform the NA to estimate to $G_{\mathrm{c}}$ and $B_{\mathrm{c}}$ (or $G_{\mathrm{s}}$ and $B_{\mathrm{s}}$ ) from the azimuthally anisotropic part of Rayleigh wave dispersion data, $a_{\mathrm{c}}(\omega, M)\left(\right.$ or $\left.a_{\mathrm{c}}(\omega, M)\right)$, using the perturbation Eq. (6) with the elastic parameters (e.g., $L \& A$ ) obtained from Step 1. (Note: there is no direct forward calculation method available to compute $a_{\mathrm{c}, \mathrm{s}}(\omega, M)$ from an azimuthally anisotropic model).

The objective of Step 1, which gives an optimal 1-D isotropic model, allows for more accurate calculation of sensitivity kernels in (6) for the subsequent estimation of the azimuthally anisotropic parameters $\left(G_{\mathrm{s}, \mathrm{c}}\right.$ and $\left.B_{\mathrm{s}, \mathrm{c}}\right)$ in Step 2 .

For a layered model ( $K$ layers) and $N_{\mathrm{d}}$ data measurements (i.e., phase velocity measurements at $N_{\mathrm{d}}$ different frequencies), Eq. (6) can be rewritten as

$\hat{a}_{\mathrm{c}, \mathrm{s}}\left(\omega_{j}\right) \approx \sum_{i=1}^{K}\left\{\frac{\delta c_{\mathrm{R}}\left(\omega_{j}\right)}{\delta A^{(i)}} B_{\mathrm{c}, \mathrm{s}}^{(i)}+\frac{\delta c_{\mathrm{R}}\left(\omega_{j}\right)}{\delta L^{(i)}} G_{\mathrm{c}, \mathrm{s}}^{(i)}\right\}$

or

$\hat{a}_{\mathrm{c}, \mathrm{s}}\left(\omega_{j}\right) \approx \sum_{i=1}^{K}\left\{\left(A^{(i)} \frac{\delta c_{\mathrm{R}}\left(\omega_{j}\right)}{\delta A^{(i)}}\right) \frac{B_{\mathrm{c}, \mathrm{s}}^{(i)}}{A^{(i)}}+\left(L^{(i)} \frac{\delta c_{\mathrm{R}}\left(\omega_{j}\right)}{\delta L^{(i)}}\right) \frac{G_{\mathrm{c}, \mathrm{s}}^{(i)}}{L^{(i)}}\right\}$, 
where $\omega_{j}$ is the $j$ th frequency $\left(j=1,2, \ldots, N_{\mathrm{d}}\right), \hat{a}_{\mathrm{c}, \mathrm{s}}\left(\omega_{j}\right)$ are the predicted data at frequency $\omega_{j}, \delta c_{\mathrm{R}}\left(\omega_{j}\right)$ is the perturbation of the phase velocity at frequency $\omega_{j}, \delta L^{(i)}$ (or $\delta A^{(i)}$ ) is the perturbation of $L$ (or $A$ ) of the $i$ th layer, and $G_{\mathrm{c}, \mathrm{s}}^{(i)}$ and $B_{\mathrm{c}, \mathrm{s}}^{(i)}$ are the azimuthally anisotropic parameters of the $i$ th layer. If there exists some simple relationship between the perturbation (in percent) of $B_{\mathrm{c}}^{(i)}$ (or $B_{\mathrm{s}}^{(i)}$ ) and the perturbation of $G_{\mathrm{c}}^{(i)}\left(\right.$ or $\left.G_{\mathrm{s}}^{(i)}\right)$ as

$\frac{B_{\mathrm{c}, \mathrm{s}}^{(i)}}{A^{(i)}}=\gamma^{(i)} \frac{G_{\mathrm{c}, \mathrm{s}}^{(i)}}{L^{(i)}}=\gamma^{(i)} \hat{G}_{\mathrm{c}, \mathrm{s}}^{(i)}$,

where $\gamma^{(i)}=\left[B_{\mathrm{c}, \mathrm{S}}^{(i)} / A^{(i)}\right] /\left[G_{\mathrm{c}, \mathrm{S}}^{(i)} / L^{(i)}\right]$ is a constant and $\hat{G}_{\mathrm{c}, \mathrm{s}}^{(i)}=G_{\mathrm{c}, \mathrm{s}}^{(i)} / L^{(i)}$, Eq. (8) becomes

$\hat{a}_{\mathrm{c}, \mathrm{s}}\left(\omega_{j}\right) \approx \sum_{i=1}^{K}\left(\gamma^{(i)} A^{(i)} \frac{\delta c_{\mathrm{R}}\left(\omega_{j}\right)}{\delta A^{(i)}}+L^{(i)} \frac{\delta c_{\mathrm{R}}\left(\omega_{j}\right)}{\delta L^{(i)}}\right) \hat{G}_{\mathrm{c}, \mathrm{s}}^{(i)}$

The sensitivity kernels $\frac{\delta c_{\mathrm{R}}\left(\omega_{j}\right)}{\delta A^{(i)}}$ and $\frac{\delta c_{\mathrm{R}}\left(\omega_{j}\right)}{\delta L^{(i)}}$ can be computed using the normal mode theory (Anderson and Dziewonski 1982). Here we compute these kernels using a difference method. In Eq. (10), if the density variation is ignored for each layer, the perturbation of $A$ and $L$ can be obtained as

$\left\{\begin{array}{l}\delta A^{(i)} \approx 2 \rho V_{\mathrm{PH}}^{(i)} \delta V_{\mathrm{PH}}^{(i)} \\ \delta L^{(i)} \approx 2 \rho V_{\mathrm{SV}}^{(i)} \delta V_{\mathrm{SV}}^{(i)}\end{array}\right.$.

So for a given velocity model, we perturb $V_{\mathrm{PH}}$ (or $V_{\mathrm{SV}}$ ) of the $i$ th layer to obtain $\delta A^{(i)}$ (or $\delta L^{(i)}$ ), and then calculate the phase velocity perturbations $\delta c_{\mathrm{R}}\left(\omega_{j}\right)$ by performing forward dispersion calculations. Thus, the sensitivity matrix $\frac{\delta c_{\mathrm{R}}\left(\omega_{j}\right)}{\delta A^{(i)}}$ or $\frac{\delta c_{\mathrm{R}}\left(\omega_{j}\right)}{\delta L^{(i)}}$ is constructed using a simple difference method.

Figure 1 shows an example of a 1-D spherical Earth velocity model and the corresponding Rayleigh wave fundamental mode phase velocity sensitivity kernels $\left(\partial c_{\mathrm{R}} /\right.$ $\partial L$ and $\left.\partial c_{\mathrm{R}} / \partial A\right)$ at 20,60 , and $100 \mathrm{~s}$. Figure 2 shows the sensitivity kernel image in the period range of 10-125 s with a period interval of $5 \mathrm{~s}$. It is evident that $c_{\mathrm{R}}$ is mostly sensitive to $L$ (or $V_{\mathrm{SV}}$ ) at depths around $1 / 3$ wavelength. Although $c_{\mathrm{R}}$ has little sensitivity to $A$ (or $V_{\mathrm{PH}}$ ) of the upper mantle, it still has considerably large sensitivity to $A$ (or $\left.V_{\mathrm{PH}}\right)$ of the upper and middle crust.

The misfit between the predicted and observed data for azimuthal anisotropy in the NA is defined as

$\Phi_{\mathrm{c}, \mathrm{s}}=\sqrt{\frac{1}{N_{\mathrm{d}}} \sum_{j=1}^{N_{\mathrm{d}}}\left(\frac{\hat{a}_{\mathrm{c}, \mathrm{s}}\left(\omega_{j}\right)-a_{\mathrm{c}, \mathrm{s}}\left(\omega_{j}\right)}{\sigma_{\mathrm{c}, \mathrm{s}}\left(\omega_{j}\right)}\right)^{2}}$,

where $\sigma_{\mathrm{c}, \mathrm{s}}\left(\omega_{j}\right)$ are the standard error of the observed data $a_{\mathrm{c}, \mathrm{s}}\left(\omega_{j}\right)$, respectively, which are obtained from surface
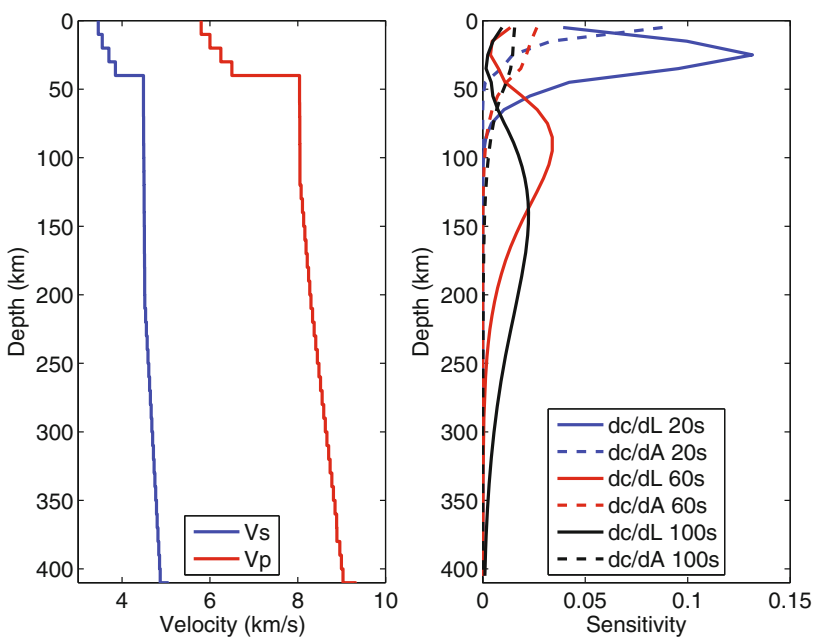

Fig. 1 1-D velocity model (left) and its corresponding sensitivity kernels for $\partial c_{\mathrm{R}} / \partial L$ and $\partial c_{\mathrm{R}} / \partial A$ at different periods (right)

wave tomographic inversion (Montagner 1986; Yao et al. 2010).

The azimuthally anisotropic wavespeed of vertically polarized shear wave can be expressed as

$\hat{\beta}_{\mathrm{SV}} \approx \sqrt{\frac{L+G_{\mathrm{c}} \cos 2 \psi+G_{\mathrm{S}} \sin 2 \psi}{\rho} .}$

Since $G_{\mathrm{c}, \mathrm{s}}$ is typically much smaller than $L$, that is, $G_{\mathrm{c}, \mathrm{s}} /$ $L \ll 1$, (13) can be approximated as

$$
\begin{aligned}
\hat{\beta}_{\mathrm{SV}} & \approx V_{\mathrm{SV}}\left(1+\frac{G_{\mathrm{c}}}{2 L} \cos 2 \psi+\frac{G_{\mathrm{s}}}{2 L} \sin 2 \psi\right) \\
& =V_{\mathrm{SV}}\left[1+\Lambda_{\mathrm{SV}} \cos 2\left(\psi-\phi_{\mathrm{F}}\right)\right]
\end{aligned}
$$

where $\Lambda_{\mathrm{SV}}$ and $\phi_{\mathrm{F}}$ are the magnitude of azimuthal anisotropy (in percent) of $V_{\mathrm{SV}}$ and the azimuth angle of the fast polarization axis, respectively, which are given by

$\Lambda_{\mathrm{SV}}=0.5 \sqrt{\hat{G}_{\mathrm{c}}^{2}+\hat{G}_{\mathrm{s}}^{2}}$,

$\Phi_{\mathrm{F}}=0.5 \tan ^{-1}\left(\hat{G}_{\mathrm{s}} / \hat{G}_{\mathrm{c}}\right)$.

\section{Application to SE Tibet}

Yao et al. (2010) investigated the depth-dependent shear wavespeed and azimuthal anisotropy in the lithosphere of SE Tibet. They used the NA to invert for the isotropic shear wavespeed model in the crust and upper mantle from the isotropic Rayleigh-wave phase velocity dispersion data in the period band 10-150 s at each grid point, which is the same as Step 1 of this study. Then they used the linearized inversion method by Montagner and Nataf (1986) (see Eq. (4)) to invert the dispersion data with azimuthal 

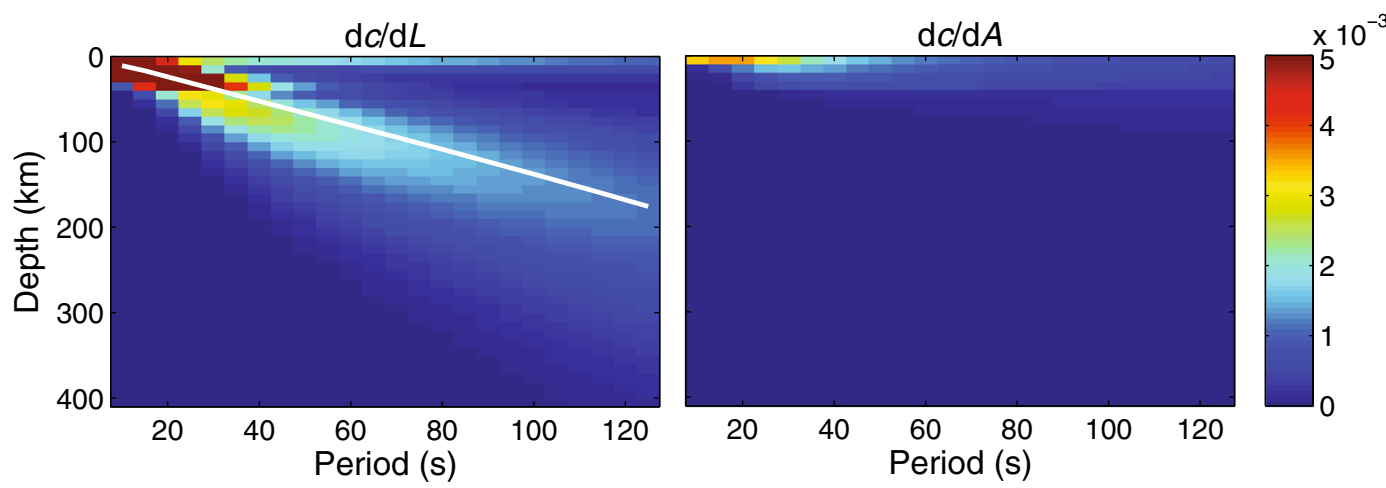

Fig. 2 Sensitivity kernel images of $\partial c_{R} / \partial L$ and $\partial c_{R} / \partial A$ in the period range of $10-125 \mathrm{~s}$. The color bar shows the value of sensitivity. The white line in the left plot gives depths of $1 / 3$ wavelength of the Rayleigh wave fundamental mode

anisotropy simultaneously for isotropic and azimuthally anisotropic shear wavespeeds at depths. Similar approaches have been taken by Lin et al. (2010) to invert for layered anisotropic model in the western US.

For the proposed two-step approach to invert for depthdependent shear wavespeed azimuthal anisotropy, we have first validated our method using the synthetic data. We construct a 1-D layered velocity model with varying magnitudes and fast axes of shear wavespeed azimuthal anisotropy in the crust and upper mantle. Since dominant anisotropic minerals, e.g., olivine in the upper mantle as well as mica and amphibole-rich minerals in the crust, will tend to result in similar fast polarization axes of $\mathrm{P}$ - and $\mathrm{S}$-waves as well as similar ratios of $\hat{B}_{c, s}^{(i)}=B_{c, s}^{(i)} / A^{(i)}$ and $\hat{G}_{\mathrm{c}, \mathrm{s}}^{(i)}=$ $G_{\mathrm{c}, \mathrm{s}}^{(i)} / L^{(i)}$ (Barruol and Kern 1996; Montagner and Nataf 1986), we set $\gamma^{(i)}=\hat{B}_{\mathrm{c}, \mathrm{s}}^{(i)} / \hat{G}_{\mathrm{c}, \mathrm{s}}^{(i)}=1$ for each layer for simplicity, similarly as Lin and Ritzwoller (2011). Then we compute the isotropic part Rayleigh-wave phase velocity dispersion (Herrmann and Ammon 2004) and azimuthally anisotropic terms $a_{\mathrm{c}, \mathrm{s}}(\omega)$ using Eq. (6) or (7). We follow Step 1 in our proposed procedure to obtain the optimal isotropic velocity model from the NA and then follow Step 2 to obtain the $\hat{G}_{\mathrm{c}, \mathrm{s}}^{(i)}$ and $\hat{B}_{\mathrm{c}, \mathrm{s}}^{(i)}$. Our tests show that the inversion results of $\hat{G}_{\mathrm{c}, \mathrm{s}}^{(i)}$ are quite accurate if we only invert for $\hat{G}_{\mathrm{c}, \mathrm{s}}^{(i)}$ with $\gamma^{(i)}$ set to be 1 . If we simultaneously estimate $\hat{G}_{\mathrm{c}, \mathrm{s}}^{(i)}$ and $\hat{B}_{\mathrm{c}, \mathrm{s}}^{(i)}$ without any constraints on their ratios, the inversion results of some model parameters will deviate from the true values and also have larger uncertainties due to trade-offs between different model parameters. This is quite similar to surface wave dispersion inversion for velocity structures. Since it is difficult to constrain $v \mathrm{p}$ structures only from dispersion data, we usually only invert for $v$ s structures from dispersion data but relating $v \mathrm{p}$ (also density) to $v$ s using some empirical relationships in the inversion (e.g., Yao et al. 2008).

Then we choose the azimuthally anisotropic phase velocity dispersion data of Rayleigh waves at the grid point $\left(101.5^{\circ}, 28.5^{\circ}\right)$ from Yao et al. (2010) to illustrate the details of the proposed two-step procedure. In the first step, the isotropic part dispersion in the period band 10-150 s (Fig. 3a) is used to invert for the isotropic shear wavespeed model (with $v \mathrm{p}$ and density related to $v \mathrm{~s}$ ) using the NA following the procedure by Yao et al. (2008). Here the Moho depth is fixed in the inversion with its value $(54 \mathrm{~km})$ approximately inferred from the receiver function analysis by $\mathrm{Xu}$ et al. (2007). We have eight parameters to be estimated, that is, shear wavespeed perturbations of the three crustal layers and five upper mantle layers with respect to a reference model. The final obtained isotropic shear wavespeed model is the posterior mean model (black line in Fig. 3b) from the Bayesian analysis of the model ensemble generated by the neighborhood search.

In the second step, we use the observed data of phase velocity azimuthal anisotropy $a_{\mathrm{c}, \mathrm{s}}\left(\omega_{j}\right)$ as well as their standard error $\sigma_{\mathrm{c}, \mathrm{s}}\left(\omega_{j}\right)$ at the same grid point (Yao et al. 2010) to estimate depth-dependent azimuthally anisotropic parameters $G_{\mathrm{s}, \mathrm{c}}\left(\right.$ and $\left.B_{\mathrm{s}, \mathrm{c}}\right)$. Due to worse azimuthal ray path
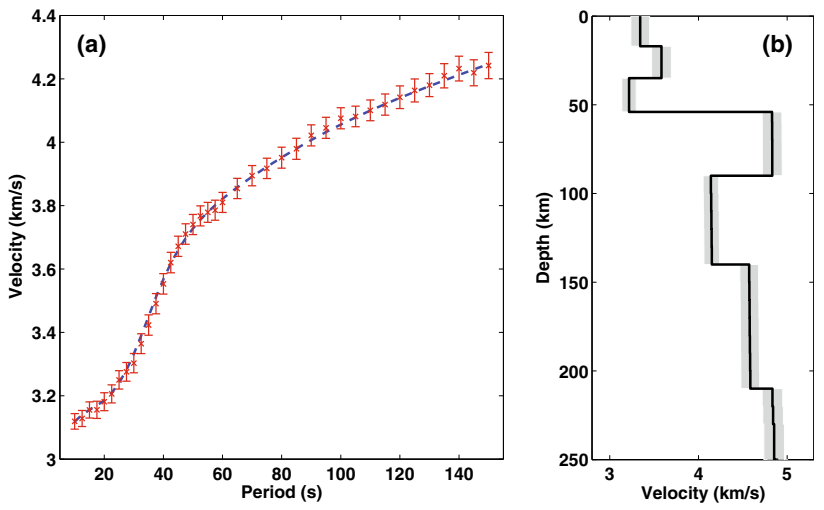

Fig. 3 a The observed isotropic part of the Rayleigh-wave phase velocity dispersion curve in SE Tibet with standard errors (red x with bars) (Yao et al. 2010) and the predicted dispersion curve (dashed blue line) of (b) the obtained posterior mean shear wavepseed model (black line) from the NA. The gray area in (b) gives the range of model standard errors 
coverage at periods above $100 \mathrm{~s}$, we only use data in the period band $10-100 \mathrm{~s}$ in this step. First, we need to compute the sensitivity kernels $\partial c_{R} / \partial L$ and $\partial c_{R} / \partial A$ from the obtained isotropic model (Fig. 3b). Here we choose $\gamma^{(i)}=$ $\hat{B}_{\mathrm{c}, \mathrm{s}}^{(i)} / \hat{G}_{\mathrm{c}, \mathrm{s}}^{(i)}=1$ for all layers. Depth-dependent $G_{\mathrm{c}}$ and $G_{\mathrm{s}}$ are separately estimated from the observed period-dependent $a_{\mathrm{c}}$ (blue dashed line in Fig. 4) and $a_{\mathrm{s}}$ (red dashed line in Fig. 4) using the NA, respectively, with the misfit function defined as Eq. (12). Here, we estimate $G_{\mathrm{c}, \mathrm{s}}$ in the six depth ranges: upper crust $(0-17 \mathrm{~km})$, middle crust $(17-35 \mathrm{~km})$, lower crust (35-54 km), and three upper mantle layers (54$90 \mathrm{~km}, 90-140 \mathrm{~km}, 140-210 \mathrm{~km})$. Figure 5 shows the posterior mean value (black line) and the corresponding standard error (shaded area) of each $G_{\mathrm{c}, \mathrm{S}} / L$ parameter from the 1-D PPDFs (Fig. 6). It appears that most 1-D PPDFs for $\hat{G}_{\mathrm{c}, \mathrm{s}}^{(i)}=G_{\mathrm{c}, \mathrm{s}}^{(i)} / L^{(i)}$ (Fig. 6) show a Gaussian distribution, and the 1-D PPDFs for $G_{\mathrm{c}} / L$ are systematically narrower than those for $G_{\mathrm{S}} / L$, indicating a smaller standard error of $G_{\mathrm{c}} / L$ than that of $G_{\mathrm{s}} / L$ (see also Fig. 5). This is probably due to the oscillating feature of $a_{\mathrm{s}}$ (Fig. 4) that are used for estimating $G_{\mathrm{s}} / L$. Figure 7 shows some examples of 2-D PPDFs that are usually used to quantify the trade-offs between different model parameters. For this particular example, the correlation between $G_{\mathrm{c}} / L$ (or $G_{\mathrm{s}} / L$ ) of two nearby depth ranges seems small as indicated by the relatively circular shape of the 2-D confidence levels.

Using the predicted $a_{\mathrm{c}, \mathrm{s}}$ (Fig. 4) from the posterior mean model (Fig. 5), we can compute the magnitude of the Rayleigh wave azimuthal anisotropy and azimuth of the

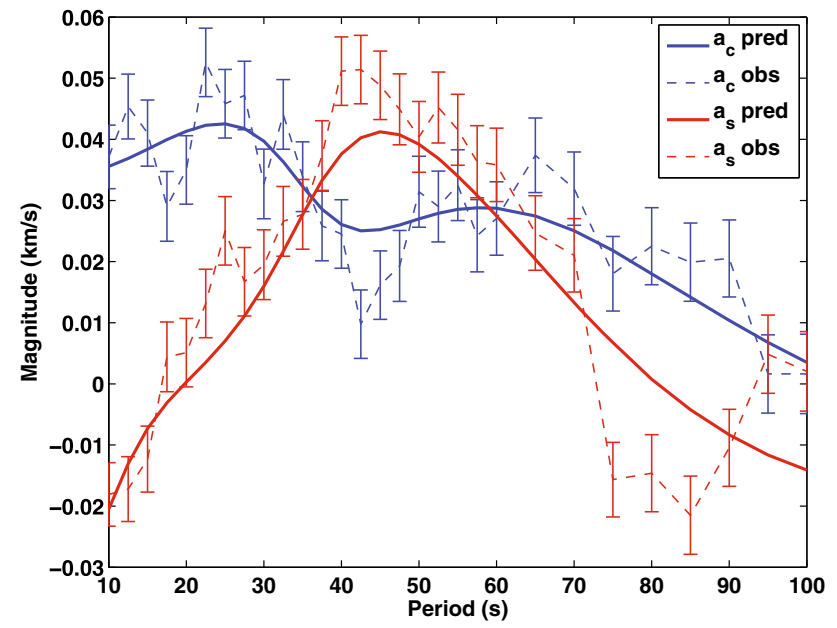

Fig. 4 The observed phase velocity azimuthal anisotropy terms $a_{\mathrm{c}}$ (dashed blue) and $a_{\mathrm{s}}$ (dashed red) and the predicted $a_{\mathrm{c}}$ (solid blue) and $a_{\mathrm{s}}$ (solid red) from the posterior mean model in Fig. 5. The error bar in red and blue shows the standard errors of the observed $a_{\mathrm{c}}$ and $a_{\mathrm{s}}$, respectively, which are obtained from the phase velocity tomography (Yao et al. 2010)
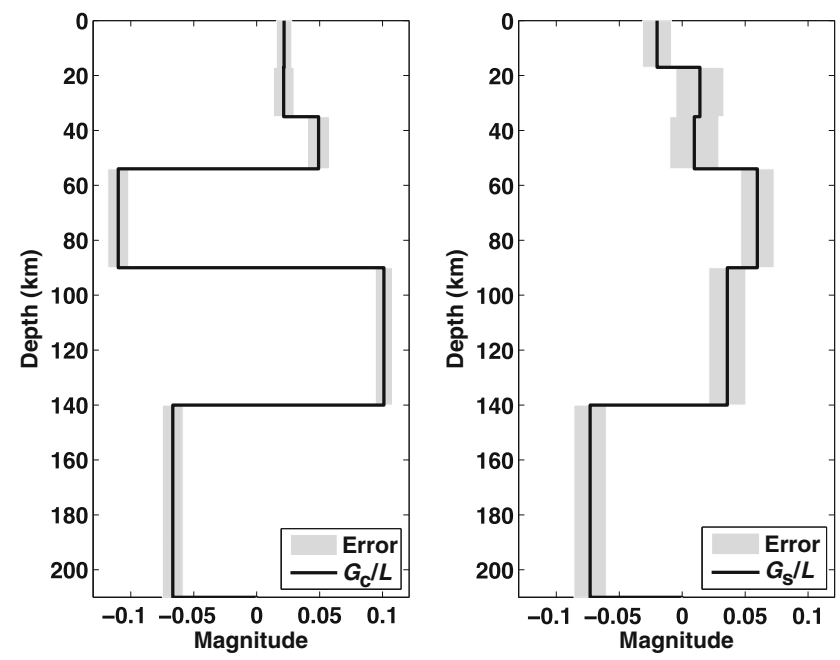

Fig. 5 The posterior mean model (black line) and its standard error (shaded area) of $\hat{G}_{\mathrm{c}}=G_{\mathrm{c}} / L($ left $)$ and $\hat{G}_{\mathrm{s}}=G_{\mathrm{s}} / L$ (right) from the $1 \mathrm{D}$ PPDFs in Fig. 6 using the NA

fast polarization axis at each period, similar as the Eqs. $(15,16)$, and compare with the observed ones as shown in Fig. 8a. The fitting is quite good in the period range of $10-70 \mathrm{~s}$ with dominant sensitivity to shear wavespeed structures up to about $150 \mathrm{~km}$. The $G_{\mathrm{c}, \mathrm{s}} / L$ in the depth range of 140-210 km estimated from the NA may have large uncertainties due to worse fitting of the data in the period band of 75-100 s. Finally, Fig. $8 \mathrm{~b}$ shows the depth-dependent magnitude and fast polarization azimuth of shear wavespeed azimuthal anisotropy using the Eqs. $(15,16)$. In our example, the magnitude of shear wavespeed azimuthal anisotropy in the crust is smaller (2\%-3\%) with nearly $\mathrm{N}-\mathrm{S}$ fast polarization axes, probably due to the deformation caused by the southward expansion of the Tibetan crustal material in SW China (Zhang et al. 2004; Royden et al. 2008). However, the uppermost mantle layer $(54-90 \mathrm{~km})$ exhibits a large magnitude of azimuthal anisotropy $(\sim 6 \%)$ with the fast polarization axis in the ENE-WSW direction, which is quite different from the pattern of crust azimuthal anisotropy. In the depth range of $90-140 \mathrm{~km}$ in the upper mantle, the shear wavespeed is very low $(\sim 0.4 \mathrm{~km} / \mathrm{s}$ lower than the global average) and the magnitude of azimuthal anisotropy is also large $(\sim 5 \%)$. However, there exists significant azimuth difference of the fast polarization axes in the uppermost mantle layer $(54-90 \mathrm{~km})$ and the underlying layer $(90-140 \mathrm{~km}$ ) that has much lower shear rigidity. Our results indicate that there could exist large differences of shear wavespeed azimuthal anisotropy in the crust and upper mantle in SE Tibet, reflecting complicated deformation patterns in this region (e.g., Yao et al. 2010; Yao 2012; Shi et al. 2012; Sun et al. 2012; Chen et al. 2013). 

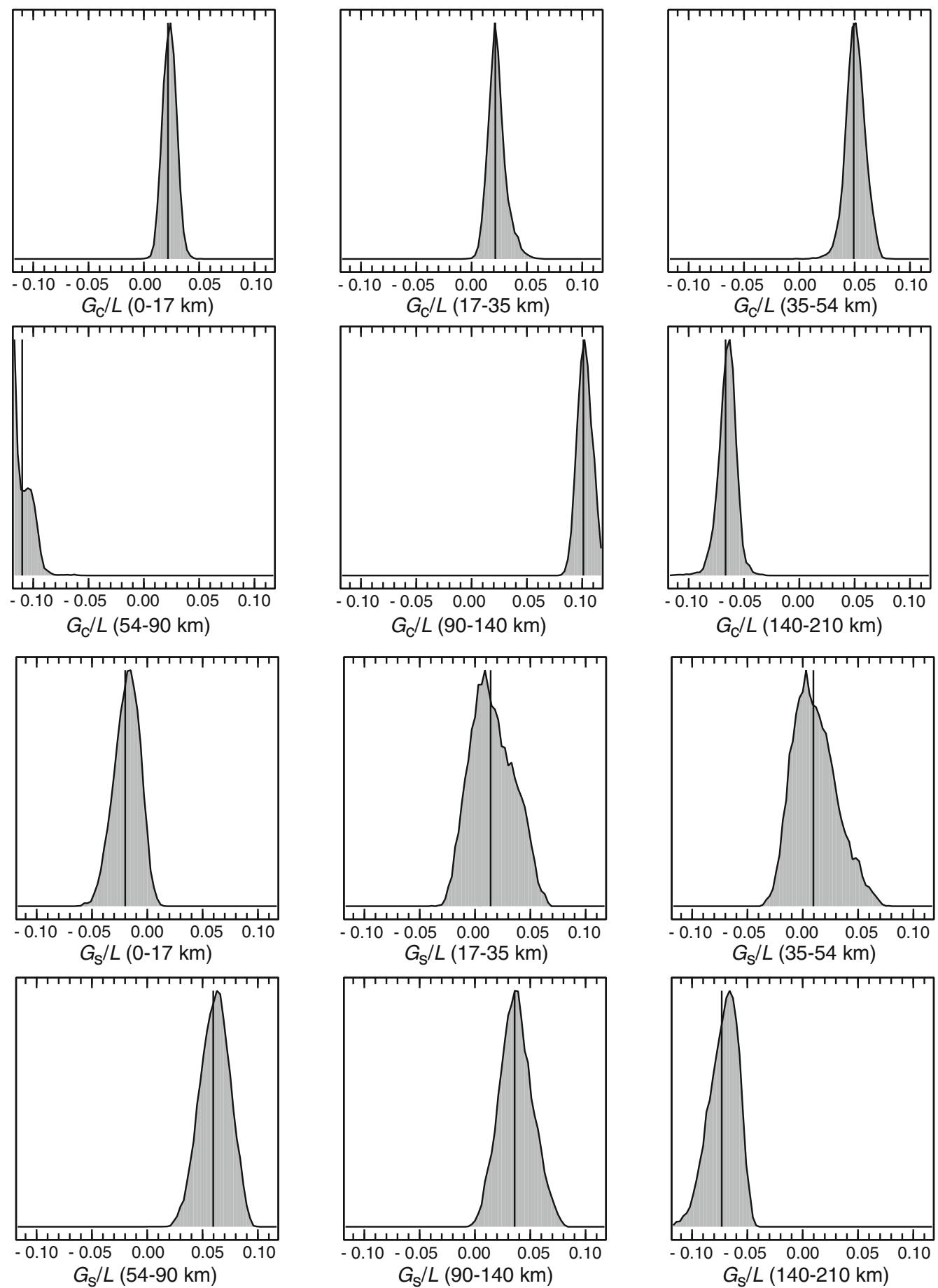

Fig. 6 The 1-D PPDFs (shaded area) for each $\hat{G}_{\mathrm{c}}=G_{\mathrm{c}} / L$ or $\hat{G}_{\mathrm{s}}=G_{\mathrm{s}} / L$ parameter in a certain depth range from the NA. The black line in each plot indicates the posterior mean value of each parameter

\section{Discussion and conclusions}

In this study, we propose a two-step approach using the NA for the point-wise inversion of depth-dependent shear wavespeeds and azimuthal anisotropy from Rayleigh wave azimuthally anisotropic dispersion data. Based on the well- constrained isotropic velocity model obtained from the isotropic dispersion data, we take a difference scheme to compute approximate Rayleigh-wave phase velocity sensitivity kernels to azimuthally anisotropic parameters $G_{\mathrm{c}, \mathrm{s}}$ and $B_{\mathrm{c}, \mathrm{s}}$. The use of the global search NA and Bayesian analysis (Sambridge 1999a, b) allows for more reliable 

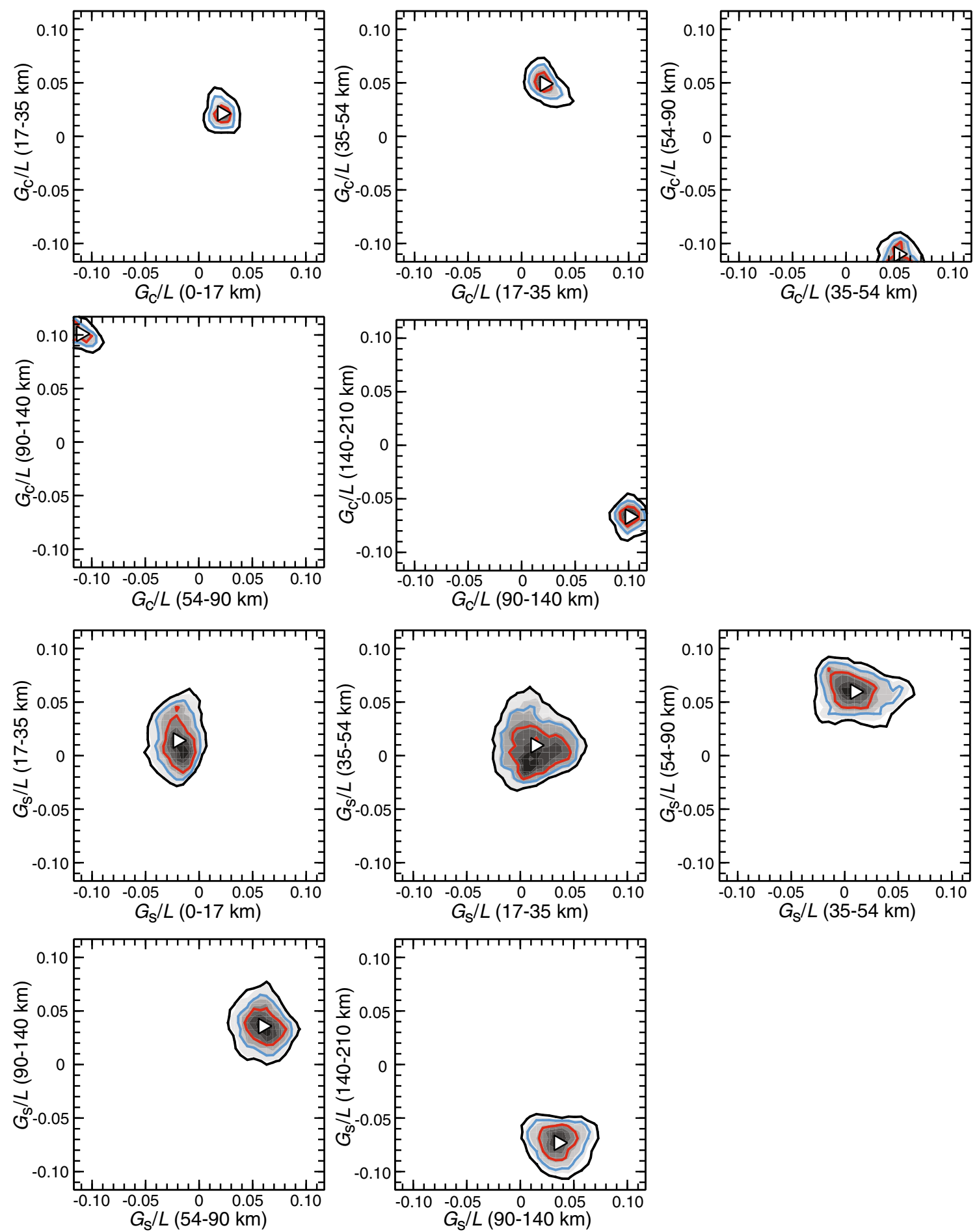

Fig. 7 The 2-D PPDFs (shaded area) from the NA for two different $\hat{G}_{\mathrm{c}}=G_{\mathrm{c}} / L$ (or $\hat{G}_{\mathrm{s}}=G_{\mathrm{s}} / L$ ) parameters. The black, blue, and red lines give the $99 \%, 90 \%$, and $60 \%$ confidence levels, respectively. The white triangle in each plot gives the posterior mean model in Fig. 6

estimates of depth-dependent shear wavespeeds and azimuthal anisotropy as well as their uncertainties.

We compare the results from this two-step global optimization approach with those from the traditional linearized inversion approach (Yao et al. 2010) in Fig. 8. Both methods show very similar directions of fast axes between 0 and $150 \mathrm{~km}$ at depths; however, the magnitude of azimuthal anisotropy shows some differences, in particular in the upper mantle. In the linearized inversion approach, Yao et al. (2010) imposed vertical smoothing and damping to 

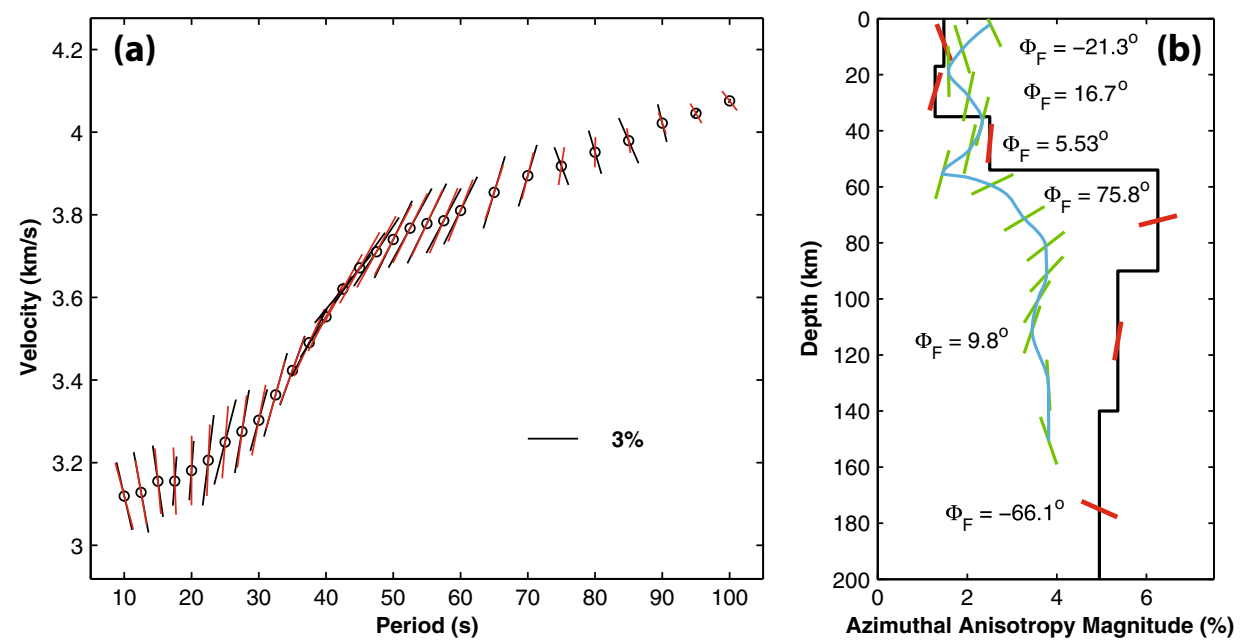

Fig. 8 a The observed (black bar) and the predicted (red bar) period-dependent phase velocity azimuthal anisotropy. The open circle gives the isotropic phase velocity. Bars in a vertical (or horizontal) direction indicate a N-S (or E-W) fast polarization axis for Rayleigh wave propagation. b Depth-dependent shear wavespeed azimuthal anisotropy obtain from the NA: the black line for the magnitude of azimuthal anisotropy $\left(\Lambda_{\mathrm{SV}}\right)$ and the red bars for the direction of fast axes $\left(\Phi_{\mathrm{F}}\right)$ in each layer (vertical for a N-S direction and horizontal for a $\mathrm{E}-\mathrm{W}$ direction). The number beside the red bar gives the azimuth angle (with respect to north) of the fast axis $\left(\Phi_{\mathrm{F}}\right)$. For comparison, the blue line and the green bars show the linearized inversion results of $\Lambda_{\mathrm{SV}}$ and $\Phi_{\mathrm{F}}$, respectively, from Yao et al. (2010)

stabilize the inversion results (Montagner and Nataf 1986). The vertical smoothing (or correlation) length is $20 \mathrm{~km}$ at the surface and gradually increases to about $35 \mathrm{~km}$ at $200 \mathrm{~km}$ depth (Yao et al. 2010). However, in the NA approach, we only fit the observed data and do not impose any model regularization terms in the misfit function (Eq. 12). This may explain the fact that the recovered magnitude of azimuthal anisotropy from our approach is larger than that from the linearized inversion approach in the upper mantle. However, both methods show that the upper mantle azimuthal anisotropy is stronger than that in the crust. Since we ignore the $\partial c_{R} / \partial F$ terms in the forward problem, this may also introduce some differences of the inversion results.

In the proposed approach, we invert for $G_{\mathrm{c}}$ and $G_{\mathrm{s}}$ separately using the misfit function defined in Eq. (12). We can also invert for $G_{\mathrm{c}}$ and $G_{\mathrm{s}}$ simultaneously by defining a new misfit function $\Phi=\Phi_{\mathrm{c}}+\Phi_{\mathrm{s}}$. With this new misfit function, we have investigated the inversion results and the model standard errors from the NA using synthetic data. Our results show that some of the model parameters are not well estimated from the NA and have larger uncertainties compared to the separated inversion scheme. This is probably because the number of model parameters has been doubled in the simultaneous inversion approach, therefore introducing more trade-offs between different parameters. So it is more reliable to invert for $G_{\mathrm{c}}$ and $G_{\mathrm{s}}$ separately.

Surface waves can provide better depth-dependent azimuthal anisotropy (Yao et al. 2010; Lin and Ritzwoller 2011) than shear wave splitting measurements in the crust and upper mantle (Savage 1999; Wang et al. 2008). Therefore, it may provide more reliable constraints on crust and upper mantle deformation patterns by examining radial variations of azimuthal anisotropy. Modeling of receiver functions can give constraints on layered anisotropy in the crust (e.g., Ozacar and Zandt 2004; Levin et al. 2008) although this approach is still very challenging in real practice. The Moho converted $\mathrm{P}_{\mathrm{m}}$ s phase splitting analysis from receiver functions can also provide constraints on average crustal azimuthal anisotropy, for instance, in SE Tibet (Xu et al. 2006; Sun et al. 2012; Chen et al. 2013; Sun et al. 2013). However, there still exists considerable inconsistency among these results. For example, Chen et al. (2013) and Sun et al. (2013) found that the splitting time of the $\mathrm{P}_{\mathrm{m}} \mathrm{s}$ wave is smaller than $0.3 \mathrm{~s}$ at most stations in SE Tibet. However, Sun et al. (2012) used a more comprehensive analysis method and found $0.5-0.9 \mathrm{~s}$ splitting time of the $\mathrm{P}_{\mathrm{m}} \mathrm{s}$ wave at a few stations in regions with thick crust in SE Tibet. Therefore, results of crustal azimuthal anisotropy from receiver function analysis may still have some uncertainties due to the use of different methods and data selection criteria.

Montagner et al. (2000) derives formulas to compute the shear wave splitting time and fast axes from depthdependent $G_{\mathrm{c}, \mathrm{s}}$ and $L$, which provides a direct link between shear wave splitting measurements and shear wavespeed azimuthal anisotropy from surface wave data. For instance, Yao et al. (2010) computed the contribution of shear wave splitting from crustal azimuthal anisotropy obtained from surface wave data and found that the thick crust in SE Tibet may contribute almost $1 \mathrm{~s}$ splitting time, which is close to 
the observed shear wave splitting time in that region. Their results suggest that the contribution of crustal anisotropy to shear wave splitting may be significant in SE Tibet, similarly as inferred from the receiver function anisotropy analysis (Sun et al. 2012). However, shear wave splitting analysis from crustal earthquakes in this region (Shi et al. 2012; Chang et al. 2014) indicates much smaller crustal azimuthal anisotropy (splitting time about $0.01-0.03 \mathrm{~s}$ per $10 \mathrm{~km})$. Although different methods have their uncertainties in estimation of crustal azimuthal anisotropy, it is still very puzzling that there exist large differences on the estimated splitting time from crustal anisotropy in different studies. Therefore, in the future it is very important to integrate different datasets together, for instance, anisotropic dispersion data, receiver functions, and shear wave splitting measurements, to better constrain the depthdependent azimuthal anisotropy and deformation patterns in the crust and upper mantle in SE Tibet and other tectonically active regions in the world.

Acknowledgments We appreciate the constructive comments from the reviewer. This study is supported by National Natural Science Foundation of China (Grant number 41222028), China National Special Fund for Earthquake Scientific Research in Public Interest (Grant number 201508008), and the Fundamental Research Funds for the Central Universities (WK2080000053).

Open Access This article is distributed under the terms of the Creative Commons Attribution License which permits any use, distribution, and reproduction in any medium, provided the original author(s) and the source are credited.

\section{References}

An M, Feng M, Zhao Y (2009) Destruction of lithosphere within the north China craton inferred from surface wave tomography. Geochem Geophys Geosyst 10:Q08016. doi:10.1029/2009GC00 2562

Anderson DL, Dziewonski AM (1982) Upper mantle anisotropy: evidence from free oscillations. Geophys J R Astron Soc 69:383-404

Barruol G, Kern H (1996) Seismic anisotropy and shear-wave splitting in lower-crustal and upper-mantle rocks from the Ivrea zone experimental and calculated data. Phys Earth Planet Inter 95:175-194

Brocher TM (2005) Empirical relations between elastic wavespeeds and density in the Earth's crust. Bull Seismol Soc Am 95(6):2081-2092

Chang LJ, Ding ZF, Wang CY (2014) Variations of shear wave splitting in the 2013 Lushan $M_{\mathrm{S}} 7.0$ earthquake region. Sci China. doi:10.1007/s11430-014-4866-8

Chen Y, Zhang Z, Sun C, Badal J (2013) Crustal anisotropy from Moho converted Ps wave splitting analysis and geodynamic implications between the eastern margin of Tibet surrounding regions. Gondwana Res 24:946-957

Debayle E, Kennett BLN, Priestley K (2005) Global azimuthal anisotropy and the unique plate-motion deformation of Australia. Nature 433:509-512

Endrun B, Lebedev S, Meier T, Tirel C, Friederich W (2011) Complex layered deformation within the Aegean crust and mantle revealed by seismic anisotropy. Nat Geosci 4: 203-207

Fry B, Deschamps F, Kissling E, Stehly L, Giardini D (2010) Layered azimuthal anisotropy of Rayleigh wave phase velocities in the European Alpine lithosphere inferred ambient noise. Earth Planet Sci Lett 297:95-102

Guo Z, Gao X, Wang W, Yao Z (2012) Upper- and mid-crustal radial anisotropy beneath the central Himalaya and southern Tibet from seismic ambient noise tomography. Geophys J Int 189: $1169-1182$

Herrmann RB, Ammon CJ (2004) Surface waves, receiver functions and crustal structure. In: Computer programs in seismology, version 3. 30 [electronic] Saint Louis University, St. Louis, Mo (available at http://www.eas.slu.edu/People/RBHerrmann/CPS330.html)

Huang Z, Su W, Peng Y, Zheng Y, Li H (2003) Rayleigh wave tomography of China and adjacent regions. J Geophys Res 103(B2):ESE 4-1-ESE 4-14

Huang H, Yao H, van der Hilst RD (2010) Radial anisotropy in the crust of SE Tibet and SW China from ambient noise interferometry. Geophys Res Lett 37:L21310. doi:10.1029/2010GL044 981

Levin L, Roecker S, Graham P, Hosseini A (2008) Seismic anisotropy indicators in Western Tibet: Shear wave splitting and receiver function analysis. Tectonophysics 462:99-108

Li H, Su W, Wang CY, Huang Z (2009) Ambient noise Rayleigh wave tomography in western Sichuan and eastern Tibet. Earth Planet Sci Lett 282:201-211. doi:10.1016/j.epsl.2009.03.021

Li Y, Wu Q, Pan J, Zhang F, Xu D (2013) An upper mantle S-wave velocity model for East Asia from Rayleigh wave tomography. Earth Planet Sci Lett 377-378:367-377

Lin FC, Ritzwoller MH (2011) Helmholtz surface wave tomography for isotropic and azimuthally anisotropic structure. Geophys J Int 186:1104-1120. doi:10.1111/j.1365-246X.2011.05070.x

Lin F, Ritzwoller MH, Snieder R (2009) Eikonal tomography: surface wave tomography by phase front tracking across a regional broad-band seismic array. Geophys J Int 177(3):1091-1110

Lin FC, Ritzwoller MH, Yang Y, Moschetti MP, Fouch MJ (2010) Complex and variable crustal and uppermost mantle seismic anisotropy in the western United States. Nat Geosci 4:55-61

Lu LY, He ZQ, Ding ZF, Wang CY (2014) Azimuthal anisotropy and velocity heterogeneity of Yunnan area based on seismic ambient noise. Chin J Geophys 57(3):822-836. doi:10.6038/cjg20140312 (in Chinese with English abstract)

Luo Y, Xu Y, Yang Y (2013) Crustal radial anisotropy beneath the Dabie orogenic belt from ambient noise tomography. Geophys $\mathbf{J}$ Int 195:1149-1164

Mainprice D (2007) Seismic anisotropy of the deep Earth from a mineral and rock physics perspective. Treatise Geophys 2:427-491

Masters G, Laske G, Bolton H, Dziewonski A (2000) The relative behavior of shear velocity, bulk sound speed, and compressional velocity in the mantle: implications for chemical and thermal structure. Geophys Monogr Ser 117:63-87

Montagner J (1986) Regional three-dimensional structures using long-period surface waves. Ann Geophys 4:283-294

Montagner J-P (2007) Deep Earth structure-upper mantle structure: global isotropic and anisotropic elastic tomography. Treatise Geophys 2:559-589

Montagner JP, Griot-Pommera DA, Lavé J (2000) How to relate body wave and surface wave anisotropy?. J Geophys Res 105(B8):19015-19027. doi:10.1029/2000JB900015

Montagner J-P, Jobert N (1988) Vectorial tomography. II: application to the Indian Ocean. Geophys J R Astron Soc 94:309-344

Montagner J, Nataf H (1986) A simple method for inverting the azimuthal anisotropy of surface waves. J Geophys Res 91:511-520 
Montagner J, Tanimoto T (1990) Global anisotropy in the upper mantle inferred from the regionalization of phase velocities. J Geophys Res 95:4797-4819

Moschetti MP, Ritzwoller MH, Lin F, Yang Y (2010) Seismic evidence for widespread western-US deep-crustal deformation caused by extension. Nature 464(8):885-889

Ozacar AA, Zandt G (2004) Crustal seismic anisotropy in central Tibet: implications for deformational style and flow in the crust. Geophys Res Lett 31:L23601. doi:10.1029/2004GL021096

Royden LH, Burchfiel BK, van der Hilst RD (2008) The geological evolution of the Tibetan Plateau. Science 321:1054-1058

Sambridge M (1999a) Geophysical inversion with a neighbourhood algorithm-I. Searching a parameter space. Geophys J Int 138: 479-494

Sambridge M (1999b) Geophysical inversion with a neighbourhood algorithm-II. Appraising the ensemble. Geophys J Int 138: $727-746$

Savage MK (1999) Seismic anisotropy and mantle deformation: what have we learned from shear wave splitting? Rev Geophys 37:65-106

Shapiro NM, Ritzwoller MH (2002) Monte Carlo inversion for a global shear velocity model of the crust and upper mantle. Geophys J Int 151(1):88-105

Shi YL, Jin W (1995) Genetic algorithms inversion of lithospheric structure from surface wave dispersion. Chin J Geophys 38(2):189-198 (in Chinese with English abstract)

Shi Y, Gao Y, Su Y et al (2012) Shear-wave splitting beneath Yunnan area of Southwest China. Earthq Sci 25(1):25-34

Silveira G, Stutzmann E (2002) Anisotropic tomography of the Atlantic Ocean. Phys Earth Planet Inter 132:237-248

Simons FJ, Zielhuis A, van der Hilst RD (1999) The deep structure of the Australian continent from surface wave tomography. Lithosphere 48:17-43

Simons FJ, van der Hilst R, Montagner JP, Zielhuis A (2002) Multimode Rayleigh wave inversion for shear wave speed heterogeneity and azimuthal anisotropy of the Australian upper mantle. Geophys J Int 151:738-754

Smith ML, Dahlen FA (1973) The azimuthal dependence of Love and Rayleigh wave propagation in a slightly anisotropic medium. J Geophys Res 78:3321-3333

Su W, Wang CY, Huang ZX (2008) Azimuthal anisotropy of Rayleigh waves beneath the Tibetan plateau and adjacent areas. Sci China (Ser D) 51(12):1717-1725

Sun X, Song X, Zheng S, Yang Y, Ritzwoller MH (2010) Three dimensional shear velocity structure of the crust and upper mantle beneath China from ambient noise surface wave tomography. Earthq Sci 23:449-463. doi:10.1007/s11589-010-0744-4

Sun Y, Niu F, Liu H, Chen Y, Liu J (2012) Crustal structure and deformation of the SE Tibetan plateau revealed by receiver function data. Earth Planet Sci Lett 349-350:186-197

Sun C, Lei J, Li C, Zhang G, Zha X, Li F (2013) Crustal anisotropy beneath the Yunnan region and dynamic implications. Chin J
Geophys 56(12):4096-4105. doi:10.6038/cjg20131214 (in Chinese with English abstract)

Wang CY, Flesch LM, Silver PG, Chang LJ, Chan WW (2008) Evidence for mechanically coupled lithosphere in central Asia and resulting implication. Geology 36:363-366. doi:10.1130/ G24450A.1

Wu JP, Ming YH, Zeng YS (2001) Smooth constraint inversion technique in genetic algorithms and its application to surface wave study in the Tibetan Plateau. Acta Seismol Sin 23(1):45-53

Xu Z, Xu M-J, Wang L-S, Liu J-H, Zhong K, Tong W-W (2006) A study on crustal anisotropy using $\mathrm{P}$ to $\mathrm{S}$ converted phases of receiver functions: application to Ailaoshan-Red River fault zone. Chin J Geophys 49(2):383-393 (in Chinese with English abstract)

Xu L, Rondenay S, van der Hilst RD (2007) Structure of the crust beneath the southeastern Tibetan Plateau from teleseismic receiver functions. Phys Earth Planet Int 165:176-193. doi:10. 1016/j.pepi.2007.09.002

Yang Y, Ritzwoller MH, Zheng Y, Shen W, Levshin AL, Xie Z (2012) A synoptic view of the distribution and connectivity of the mid-crustal low velocity zone beneath Tibet. J Geophys Res 117:B04303. doi:10.1029/2011JB008810

Yao H, Beghein C, van der Hist RD (2008) Surface wave array tomography in SE Tibet from ambient seismic noise and twostation analysis: II-crustal and upper-mantle structure. Geophys J Int 163:205-219. doi:10.1111/j.1365-246X.2007.03696.x

Yao H, van der Hilst RD, Montagner JP (2010) Heterogeneity and anisotropy of the lithosphere of SE Tibet from surface wave array tomography. J Geophys Res 115:B12307. doi:10.1029/ 2009JB007142

Yao H (2012) Lithospheric structure and deformation in SE Tibet revealed by ambient noise and earthquake surface wave tomography: Recent advances and perspectives. Earthq Sci 25:371-383. doi:10.1007/s11589-012-0863-1

Yi G, Yao H, Zhu J, van der Hilst RD (2010) Lithospheric deformation of continental China from Rayleigh wave azimuthal anisotropy. Chin J Geophys 53:256-268. doi:10.3969/j.issn. 0001-5733.2010.02.004 (in Chinese with English abstract)

Zhang P, Shen Z, Wang M, Gan W, Burgmann R, Molnar P, Wang Q, Niu Z, Sun J, Wu J, Sun H, You X (2004) Continuous deformation of the Tibetan Plateau from global positioning system data. Geology 32(9):809-812

Zheng Y, Yang Y, Ritzwoller MH, Zheng X, Xiong X, Li Z (2010) Crustal structure of the northeastern Tibetan Plateau, the Ordos block and the Sichuan basin from ambient noise tomography. Earthq Sci 23:465-476. doi:10.1007/s11589-010-0745-3

Zhou Y, Nolet G, Dahlen FA, Laske G (2006) Global upper-mantle structure from finite-frequency surface-wave tomography. J Geophys Res 111:B04304. doi:10.1029/2005JB003677 